\title{
Biologic therapy for psoriasis - still searching for the best target ${ }^{*}$
}

\author{
Teresa Pinto-Almeida ${ }^{1}$
}

\author{
Tiago Torres ${ }^{1}$
}

\author{
DOI: http://dx.doi.org/10.1590/abd1806-4841.20142898
}

\begin{abstract}
Psoriasis is a chronic skin disease that results from the complex interaction between genetic and environmental factors. Over the last few decades, scientific evidence has redirected the focus of therapeutic studies to the immunologic pathways underlying its pathogenesis. This led to the biologic boom that we are currently experiencing, with the development and approval of targeted progressively more selective biological therapies and ongoing clinical trials of increasingly specific drugs, given their important implications for long-term efficacy and safety. Nevertheless, the search for the optimal biologic is still ongoing, and the best target has yet to be found.

Keywords: Biological therapy; Cytokines; Psoriasis
\end{abstract}

Psoriasis is a chronic skin disease that results from the complex interaction between genetic and environmental factors. ${ }^{1}$ Over the last few decades, scientific evidence has shown that the keratinocyte proliferation that characterizes psoriasis is triggered by activated T-cells, thus redirecting the focus of therapeutic studies to the immunologic pathways contributing to its pathogenesis. ${ }^{2}$ This led to the biologic boom that we are currently experiencing, with the development and approval of progressively more selective targeted biological therapies and ongoing clinical trials of increasingly specific drugs, given their important implications for long-term efficacy and safety.

The major pathogenic pathways involved in psoriasis that scientific research has brought to light so far comprise: the one involving interleukin (IL)-12, composed of two subunits ( $\mathrm{p} 40$ and $\mathrm{p} 35)$, driving a Thelper (Th) 1 response; the IL-23/IL-17 axis, the former composed by the $\mathrm{p} 40$ and $\mathrm{p} 19$ subunits and the later with two isoforms, $\mathrm{A}$ and $\mathrm{F}$, stimulating the expansion of Th17 and Th22 cells and production of IL-17 and IL22 - now known to have key effects on the epidermis and upregulating other proinflammatory cytokines and growth factors, therefore creating a self-amplifying inflammatory process; and tumor necrosis factor (TNF)- $\alpha$ signaling, a pleiotropic cytokine pathway that activates multiple immune cell types of both innate and adaptive immunity (Figure 1) ${ }^{1,2}$

The first biologic therapies, namely alefacept and efalizumab (withdrawn), acted by blocking Tcells, suppressing overall cytokine production and thus inhibiting the activation and proliferation of all T-cell subtypes, with a broad effect on the immune system and, consequently, wide-ranging potential side effects. ${ }^{2-4}$ This prompted unceasing investigation for more selective drugs.

TNF- $\alpha$ is a potent pro-inflammatory cytokine with significant interactions with the other pathogenic cytokines in psoriasis. Its blockade is thought to improve psoriasis due to its impact in down-regulating IL-23, Th-17 cells, and, later, Th-1 related genes. ${ }^{5}$ Etanercept, adalimumab, and infliximab are approved for psoriasis, and new drugs are currently undergoing clinical trials. As TNF- $\alpha$ plays also an important role in innate immunity, resulting in broad immunosuppression, the risk of infection and malignancy with these therapies cannot be disregarded. ${ }^{5}$

Available anti-IL-12/IL-23 therapies, such as ustekinumab, target the p40 subunits of the two cytokines. ${ }^{6}$ Both the p19 and p40 subunits of IL-23 are amplified in psoriasis, unlike IL-12p35, which is not elevated; this suggests that IL-23 might have greater

Received on 27.06.2013

Approved by the Advisory Board and accepted for publication on 29.08.2013

* Work performed at the Dermatology Department, Hospital de Santo António - Centro Hospitalar do Porto - Porto, Portugal.
Financial Support: None.

Conflict of Interests: None.

Centro Hospitalar do Porto - Hospital de Santo António - Porto, Portugal.

C 2014 by Anais Brasileiros de Dermatologia 


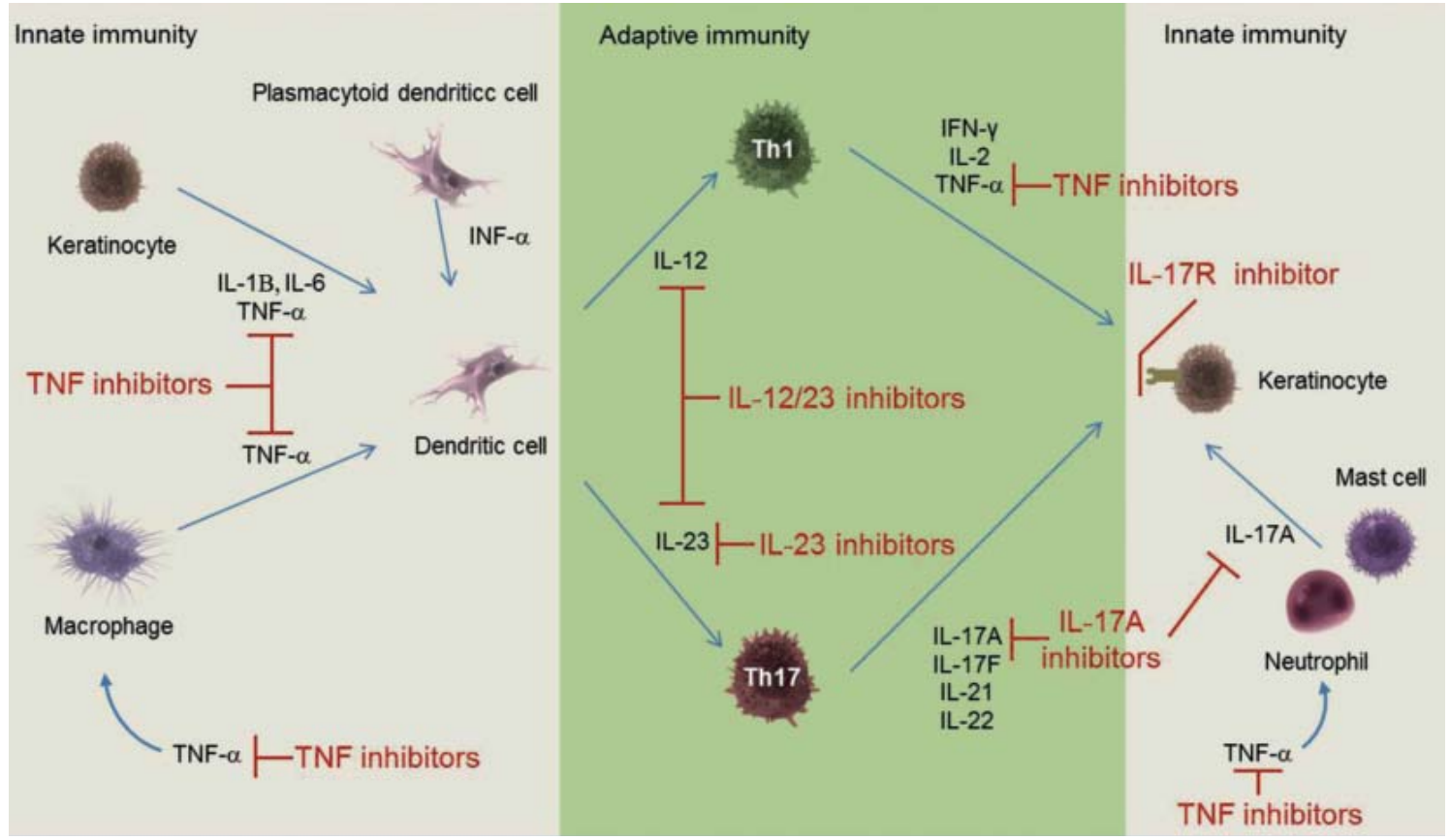

FIGURE 1: TNF- $\alpha$ inhibitors - act by blocking TNF- $\alpha$, with impact on innate and adaptive immunity. IL-12/23 inhibitors - block the generation and maintenance of Th1 and Th17 cells. IL-23 inhibitor - acts selectively on the IL23/Th17pathway, preserving the IL-12/Th1 pathway. IL-17A inhibitors - act selectively at the level of an effector cytokine, preserving other IL-17 producing cell functions. IL-17 receptor inhibitors - act at the level of a shared IL-17 cytokine receptor, blocking multiple members of the IL-17 cytokine family (IL-17A/F/C/E). Abbreviations: TNF, tumor necrosis factor; IL, interleukin; Th, T helper.

impact in psoriasis than IL-12. ${ }^{2}$ These drugs directly inhibit T-cell activation, unlike anti-TNFa preparations, which act indirectly through the inhibition of IL-23 by dendritic cells. ${ }^{2}$ Besides, the innate immunity is not directly suppressed by inhibition of p40, with a theoretically superior safety profile. Although more selective, an additional refinement regarding this inflammatory axis recently emerged, with an alternate targeting of the p19 subunit of IL-23 alone, sparing the p40 subunit and, consequently, not affecting the Th- 1 driven response. ${ }^{2}$ This new approach and inhibition of the IL-23 are currently being tested in phase II clinical trials, in order to assess the potential benefits of guselkumab and tildrakizumab. ${ }^{2}$

Regarding IL-17 inhibition, secukinumab is currently in phase III trials and ixekinumab in phase II. ${ }^{78}$ Only the IL-17A isoform is targeted; according to recent data, this approach has the advantage of maintaining a high efficacy of blockade of this major cytokine with a low risk of adverse events resulting from immunosuppression, probably due to compensatory action by the other isoform, although the clinical significance of these facts is not completely eluci- dated ${ }^{7,8}$ Nevertheless, acting selectively at the level of a key effector cytokine might preserve the other cellular immune functions, so the spectrum of immunosuppression is undoubtedly narrower than with any previous therapy, and the targeted molecules much more specific to the pathogenesis of psoriasis, making this a promising drug. IL-17 receptor blockers, such as brodalumab, are also under phase II trials, with the same potential benefits of the aforementioned agents, but inhibiting multiple members of the IL-17 family and so with a different side effect profile (which has yet to be elucidated). ${ }^{9}$

Other possible pathways are under the scrutiny of laboratory investigation, and IL-22 has appeared as a promising target. It is strictly related to the critical Th17/Th22 pathway, thus making it a smart option for the therapy of psoriasis. ${ }^{10}$ Nevertheless, trials of fezakinumab, an IL-22 antibody, were discontinued. ${ }^{10}$

In the era of biologics and with the advent of genetic and molecular research, therapy targets are becoming progressively more selective. The recognition of the major pathogenic pathways involved in psoriasis and the knowledge of their clinical implica- 
tions prompted the emergence of numerous novel therapies. The goal remains to achieve selective immunosuppression with minimal side effects, as the further downstream the target of blockade in the inflammatory cascade, the lower the resultant immunosuppression and the consequent risks of infection and malignancy. Agents once considered selective only some years ago will probably soon be replaced by more recent and more focused therapies. If we think we have come too far, much remains to be done in the future, and further large, controlled studies of recent drugs are needed to clarify their role in the complex management of psoriasis.

\section{REFERENCES}

1. Nestle FO, Kaplan DH, Barker J. Psoriasis. N Engl J Med. 2009;361:496-509.

2. Nograles KE, Krueger JG. Anti-cytokine therapies for psoriasis. Exp Cell Res. 2011;317:1293-300

3. Krueger GG, Papp KA, Stough DB, Loven KH, Gulliver WP, Ellis CN; Alefacept Clinical Study Group. A randomized, double-blind, placebo-controlled phase III study evaluating efficacy and tolerability of 2courses of alefacept in patients with chronic plaque psoriasis. J Am Acad Dermatol. 2002;47:821-33.

4. Guttman-Yassky E, Vugmeyster Y, Lowes MA, Chamian F, Kikuchi T, Kagen M, et al. Blockade of CD11a by efalizumab in psoriasis patients induces a unique state of Tcell hyporesponsiveness. J Invest Dermatol. 2008;128:1182-91.

5. Wong M, Ziring D, Korin Y, Desai S, Kim S, Lin J, et al. TNFalpha blockade in human diseases: mechanisms and future directions. Clin Immunol. 2008;126:121-36.

6. Krueger GG, Langley RG, Leonardi C, Yeilding N, Guzzo C, Wang Y, et al. A human interleukin-12/23 monoclonal antibody for the treatment of psoriasis. N Engl J Med. 2007;356:580-92.

7. Girolomoni G, Mrowietz U, Paul C. Psoriasis: rationale for targeting interleukin-17. Br J Dermatol. 2012;167:717-24.

8. Patel DD, Lee DM, Kolbinger F, Antoni C. Effect of IL-17A blockade with secukinumab in autoimmune diseases. Ann Rheum Dis. 2013;72:ii116-23.

9. Papp KA, Leonardi C, Menter A, Ortonne JP, Krueger JG, Kricorian G, et al. Brodalumab, an anti-interleukin-17-receptor-antibody for psoriasis. N Engl J Med. 2012;366:1181-9.

10. Gudjonsson JE, Johnston A, Ellis CN. Novel systemic drugs under investigation for the treatment of psoriasis. J Am Acad Dermatol. 2012;67:139-47.

\author{
MAILING ADDRESS: \\ Teresa Pinto-Almeida \\ Department of Dermatology, \\ Hospital de Santo António - Centro Hospitalar do Porto, \\ Edifício das Consultas Externas, \\ Ex. CICAP, \\ Rua D. Manuel II, s/n \\ 4100 - Porto - Portugal. \\ E-mail: teresap.almeida@hotmail.com
}

How to cite this article: Pinto-Almeida T, Torres T. Biologic therapy for psoriasis - still searching for the best target. An Bras Dermatol. 2014;89(2):365-7. 